\title{
Унифицированный подход к лечению больных с проникающими колото-резаными ранениями брюшной полости
}

\begin{abstract}
За период с 2010 по 2015 г., по данным МОЗ Украины, общий показатель смертности в Украине находится на уровне 14,516,3 \%. При этом в 6 \% всех случаев смерти причиной являлись травмы. Открытая проникающая травма живота остается одной из основных причин летальных исходов. При этом ножевые ранения встречаются почти в три раза чаще, чем огнестрельные [3]. В последние годы в мире пересмотрели подходы к ведению больных с проникающими ранениями, склонившись в сторону избирательного применения оперативного и неоперативного методов лечения [2].

Широкое распространение получил метод селективного неоперативного лечения больных с проникающими ранениями брюшной полости $[4,10,14]$. Суть данного метода заключается том, что, применяя современные диагностические методы, а также клинический мониторинг состояния больного, в определенных случаях можно обойтись без применения лапаротомии. Данная методика позволяет в 55 \% случаев ножевых ранений вести больных, не прибегая к оперативному вмешательству, сократить количество ненужных лапаротомий до 9-19 \%, уменьшить длительность пребывания больных в стационаре до 3,9 дня по сравнению с 6 при лапаротомии, а также снизить материальные затраты на лечение пациентов.

При отборе больных для неоперативного ведения лучше ориентироваться на данные общеклинических исследований (общая чувствительность и специфичность серийного клинического обследования брюшной полости составляет 87,3 и 93,5 \% соответственно) [10]. Хорошим подспорьем являются данные компьютерной томографии, однако данные чувствительности и специфичности данного метода колеблются у разных авторов от 60 до более 90 \% [4, 9, 11, 13]. Диагностическая ценность ультразвукового исследования в первые часы после полученной травмы составляет всего 46 \% [12], а обзорной рентгенографии - 2,6 \% [7]. Применение лапароскопии в диагностике и лечении колото-резаных ранений брюшной стенки является приоритетным методом в случае стабильной гемодинамики у больных [8].
\end{abstract}

Ключевые слова: селективное неоперативное ведение; ножевые ранения; брюшная полость; лапароскопия.

За период с 2010 по 2015 г., согласно данным Центра медицинской статистики МОЗ Украины, общий показатель смертности в Украине находится на уровне 14,5-16,3 \%. При этом в 6 \% всех случаев смерти причиной являлись травмы. В структуре травм открытая проникающая травма живота по прежнему остается одной из основных причин летальных исходов как в Украине, так и во всем мире. При этом ножевые ранения встречаются почти в три раза чаще, чем огнестрельные [3].

Уже много десятилетий лапаротомия является стандартом лечения проникающих ранений брюшной полости. В последние годы в мире пересмотрели подходы к ведению больных с проникающими ранениями, склонившись в сторону избирательного применения оперативного и неоперативного методов лечения [2]. Внедрение и усовершенствование диагностических методов визуализации, таких как лапароскопия, компьютерная томография, магнитно-резонансная томография, ультразвуковые методы исследования, в значительной степени способствовало развитию неоперативных методов ведения больных с проникающими ранениями брюшной полости.

В последнее десятилетие в мире широкое распространение получил метод селективного неоперативного лечения больных с проникающими ра- нениями брюшной полости. Суть данного метода заключается том, что, применяя современные диагностические методы, а также клинический мониторинг состояния больного, в определенных случаях можно обойтись без применения лапаротомии.

Отбор большинства пациентов для данного метода основывается на отсутствии у них гемодинамических нарушений и клиники перитонита $[4,10,14]$. Оба эти понятия можно считать относительными. Значение систолического артериального давления <90 мм рт. ст., ЧСС >100 уд/мин указывают на гемодинамические нарушения. Но при этом многие пациенты могут быть в состоянии шока с показателями, которые, на первый вигляд, кажутся нормальными [6]. Возраст, сопутствующая патология, применяемые лекарственные средства являются одними из факторов, которые могут повлиять на артериальное давление и частоту сердечных сокращений, а, следовательно, привести в заблуждение доктора, который полагается только на абсолютные значения. Такой же неприятный сюрприз можно ожидать и от перитонита: пожилой возраст больного, индивидуальность болевого порога каждого пациента, применение обезболивающих средств на догоспитальном этапе может существенно изменить клинику развивающейся внутрибрюшной катастрофы у больного. 
К результатам инструментальных методов исследования также нужно относиться критично, учитывая их невысокую специфичность и чувствительность в первые часы после получения травм [1, 11].

В проспективном исследовании Udofi и др. из 75 пациентов с проникающими ранениями брюшной полости у 54 оказались отрицательными результаты первичного ультразвукового исследования. При этом у 13 из 54 имелись ложноотрицательные результаты, и при дальнейшем обследовании у них были обнаружены тяжелые травмы органов брюшной полости. Это показало, что диагностическая ценность ультразвукового исследования в первые часы после полученной травмы составляет всего 46 \% [12]. Диагностическая ценность обзорной рентгенографии в первые часы с момента поступления составляет всего 2,6 \% [7].

Многие из хирургов считают, что тяжесть травмы у больного может быть довольно надежно оценена с помощью компьютерной томографии. По данным некоторых исследователей, наличие внутрибрюшной травмы может быть определено с чувствительностью и специфичностью >90\% [4, 13].

Однако в более современных исследованиях доказано, что данные компьютерной томографии не могут быть абсолютным показанием к проведению больному лапаротомии.

В исследовании Nicholson и др., включающем 93 больных с проникающими ранениями брюшной полости за период с 2005 по 2013 г., показано, что компьютерной томографии не должно отдаваться предпочтение в выборе тактики ведения больных с травматическими повреждениями брюшной полости. Основу должны составлять клинические данные. В данном исследовании у 47 (50,5 \%) больных имелись повреждения сальника, у 41 $(44,1 \%)$ имелись повреждения органов брюшной полости, и у 2 (2,2 \%) имелись повреждения органов брюшной полости и сальника. 74 (80,4 \%) пациентам была выполнена лапаротомия. 70 (65,8 \%) больным была дооперационно произведена компьютерная томография. Только в 60 \% пациентов результаты компьютерной томографии были сравнимы с интраоперационной находкой [11].

Даже наличие, по данным компьютерной томографии, внутрибрюшной свободной жидкости у больных с травмой брюшной полости не является абсолютным показанием к ургентной лапаротомии.

Об этом подчеркивается в исследовании Mahmood Ismail и др. В общей сложности 122 пациента были включены в исследование. Средний возраст пациентов составил $(33 \pm 12)$ года. 34 па- циента подверглись лапаротомии, 31 из которых имел повреждения внутренних органов. Небольшие повреждения кишечника были обнаружены у 13 больных, большие травмы кишечника - у 11 и травмы брыжейки - у 7 пациентов. У остальных 3 пациентов во время лапаротомии повреждений внутренних органов не обнаружено. Таким образом, обнаружение внутрибрюшной жидкости при компьютерной томографии является неспецифичной для прогнозирования повреждений кишечника или брыжейки кишечника и не является абсолютным показанием к хирургическому вмешательству. Тем не менее, корреляция между результатами компьютерной томографии и клиническими данными является важной для обеспечения оптимальной диагностики травм кишечника и брыжеечных травм [9].

Таким образом, компьютерная томография может быть полезным подспорьем в отборе пациентов, которым не нужна операция, однако тщательный мониторинг динамики состояния здоровья является обязательным для больных, которые подлежат консервативному лечению. Этим пациентам должны быть выполнены повторные клинические, инструментальные и лабораторные исследования в течение первых 12-24 ч после поступления в больницу.

В исследовании Navsaria Р. Н. и др., включающем 186 пациентов с проникающими ножевыми ранениями брюшной полости, было показано, что важнейшая роль в выборе тактики ведения больного должна отдаваться именно клиническим данным. По данным многократных клинических обследований больного к 112 пациентам (60,2 \%) применен метод селективного неоперативного ведения. 100 (89,3 \%) из этих пациентов были успешно пролечены без выполнения лапаротомии. Остальным 12 пациентам произведена лапаротомия с задержкой. В 2-х случаях (16,7%) во время лапаротомии повреждений внутренних органов не обнаружено. Неоперативное ведение в целом было успешным у 53,8 \% больных. Общая чувствительность и специфичность серийного клинического обследования брюшной полости составляла 87,3 и 93,5 \% соответственно [10].

Неоперативное ведение больных с ножевыми ранениями брюшной полости стало стандартом лечения в Соединенных Штатах Америки, около 55 \% от всего количества больных с ножевыми ранениями передней брюшной стенки могут вестись консервативно. Даже при наличии проникающего ранения значительное число пациентов не имеет каких-либо тяжелых внутрибрюшных травм, требующих неотлагательной операции. Это снижает количество ненужных лапаротомий до 9-19 \% [6, 10]. 
Исследование William W. Норе включает 86 пациентов с проникающими ранениями брюшной полости. 12 (14 \%) больным со стабильной гемодинамикой и без клинических признаков перитонита проводилось неоперативное лечение. Средний возраст составил 30 лет (диапазон 21-39 лет). Пациенты мужского пола составляли 92 \%. В целом, неоперативное лечение не удалось у 3 пациентов (25\%); одному выполнено дренирование абсцесса сальниковой сумки на 4-й день с момента госпитализации, второму выполнено ушивание желудка и ранения диафрагмы в день госпитализации. В третьем случае у больного развилась билома, которая требовала проведения чрескожного дренирования. У остальных больных не было осложнений. Летальных случаев среди больных не зарегистрировано. Средняя продолжительность пребывания составила 3,9 суток; 83 \% пациентов были выписаны домой [14].

Таким образом, у гемодинамически стабильных больных без клиники перитонита и данных об изолированных травмах солидных органов применение метода селективного неоперативного ведения больных с проникающими ранениями брюшной полости является безопасным. Тем не менее, данный метод может привести к задержке диагностики повреждения полых органов.

B докладе D. Demetrides и др. провели проспективное исследование 152 пациентов с проникающими травмами брюшной полости и повреждением внутренних органов. Из них 45 пациентов (29,6 \%) имели ножевые ранения. Печень была наиболее часто травмирована из паренхиматозных органов (63 \%). Далее следует травма почек $(20,3 \%)$ и селезенки $(16,7 \%)$. Сорок один пациент (27\%), в том числе 18 больных с наличием множественных (3-5) ножевых ранений, успешно обошлись без лапаротомии и не имели никаких послеоперационных осложнений со стороны брюшной полости. Пациенты с изолированными ранениями паренхиматозных органов, которые велись консервативно, имели значительно меньший срок госпитализации, чем пациенты, которые перенесли лапаротомию (в среднем 3 дня против 6 дней соответственно, $\mathrm{p}=0,002)$ [6].

В последнее время на первый план выходит применение диагностической и лечебной лапароскопии в лечении пациентов с проникающими ранениями брюшной полости. Тем не менее, многочисленные споры существуют по поводу показаний, противопоказаний и уместности использования данного метода.

В исследование Asuquo М. и др. были включены 114 пациентов с колото-резаными ранениями брюшной полости, которым проводилась лапа- роскопия. Ножевые ранения были нанесены у 81 (71\%) пациента. Коэффициент конверсии составил 7 \%. Операция была завершена лапароскопически у 79 больных с ножевыми ранениями (74,5 \%). Диагностическая лапароскопия была выполнена 44 пациентам (41,5 \%) и лапароскопическая операция - 62 пациентам (58,5 \%). В общей сложности, 13 пациентам (12 \%) выполнено повторное оперативное вмешательство после лапароскопии. Умерли 2 больных (1,9 \%). Послеоперационные осложнения не были специфическими для лапароскопии. Пропущенных травм не было зарегистрировано. Таким образом, диагностическая и лечебная лапароскопия у больных с проникающими ранениями брюшной полости является приоритетным методом в случае стабильной гемодинамики у больных. Расположение травм не является ограничивающим фактором, хотя оно влияет на размещение портов. Конверсия не является осложнением, а скорее правильным способом завершения операции в соответствующей ситуации [8].

Таким образом, в отсутствие явных признаков тяжелого повреждения органов брюшной полости, безопасным методом ведения пациентов с колото-резаными ранениями брюшной полости является селективное неоперативное лечение. Повторные общеклинические исследования являются основой для выбора тактики лечения больных, и это отмечено многими авторами. Дополнительные исследования, такие как диагностический перитонеальный лаваж, ангиография, внутривенная пиелография и компьютерная томография, применимы на индивидуальной основе, в зависимости от конкретного случая, так как пока не хватает убедительных оснований для их рутинного использования [4].

Выводы. 1. Множественные исследования показывают, что до 55 \% больных с ножевыми ранениями могут вестись, не прибегая к оперативному вмешательству. Это, в свою очередь, снижает количество ненужных лапаротомий до 9-19 \% [6, 10], а также уменьшает длительность пребывания больных в стационаре до 3,9 дня против 6 при лапаротомии и снижает материальные расходы на лечение больного $[6,14]$.

2. При отборе больных для неоперативного ведения лучше ориентироваться на данные общеклинических исследований (общая чувствительность и специфичность серийного клинического обследования брюшной полости составляет 87,3 и 93,5\% соответственно) [10].

3. Хорошим подспорьем являются данные компьютерной томографии, однако данные чувствительности и специфичности этого метода колеблются у разных авторов от 60 до более $90 \%$ 
$[4,9,11,13]$. Диагностическая ценность ультразвукового исследования в первые часы после полученной травмы составляет всего $46 \%$ [12], а обзорной рентгенографии - 2,6 \% [7].

\section{СПИСОК ЛИТЕРАТУРЫ}

1. Борисов А. Е. Возможности эндовидеохирургии при торакоабдоминальных ранениях / А. Е. Борисов, К. Г. Кубачев, А. В. Кукушкин // Эндоскопическая хирургия. - 2012. № 3. - C. 5-9.

2. Панкратов А. А. Возможность консервативного ведения закрытой и открытой травмы печени различной степени тяжести / А. А. Панкратов, И. Е. Хатьков, Р. Е. Израилов // Альманах клинической медицины. - 2015. - № 40. - С. 132-137. 3. Украинская база медико-статистической информации “Здоровье для всех” [Электронный ресурс] / Центр медицинской статистики МОЗ Украины. - 2015. - Режим доступа : http://medstat.gov.ua/ukr/normdoc.html

4. Asuquo M. Penetrating abdominal trauma: experience in a teaching hospital, calabar, Southern Nigeria / M. Asuquo, M. Umoh, V. Nwagbara // International Journal of Clinical Medicine. - 2012. - № 3. - P. 426.

5. Como JJ. Practice management guidelines for selective nonoperative management of penetrating abdominal trauma / J. J. Como, F. Bokhari, W. C. Chiu // Journal of Trauma. - 2010. - №68. - P. 21-33.

6. Demetriades D. Selective nonoperative management of penetrating abdominal solid organ injuries / D. Demetriades, P. Hadjizacharia, C. Constantinou // Annals of Surgery - 2006. № 244. - P. 620-628.

7. Demetriades D. The management of penetrating injuries of abdomen. A prospective study of 230 patients / D. Demetriades, B. Rabinowitz, C. Sofianos // Annals of Surgery - 2008. - № 207. - P. 72-74.

\section{REFERENCES}

1. Borisov, A.E., Kubachev K.G. \& Kukushkin A.V. (2012). Vozmozhnosti endovideokhirurgii pri torakoabdominalnykh raneniyakh [The possibilities of endovideosurgery in thoracoabdominal wounds]. Endoskopicheskaya khirurgia. - Endoscopic Surgery, 3, 5-9 [in Russian].

2. Pankratov, A.A., Hatkov, I.E., \& Izrailov, R.E. (2015). Vozmozhnost konservativnogo vedenia zakrytoi i otkrytoi travmy pecheni razlichnoi stepeni tiazhesti [The possibility of conservative management of closed and open injuries of the liver of varying severity]. Almanakh klinicheskoi meditsiny - Almanac of Clinical Medicine, 40, 132-137 [in Russian].

3. Ukrainskaia baza medico-statisticheskoi informatsii "Zdorovie dlia vsekh" [Ukrainian base of medical statistical information "Health for all"]. (n.d.). medstat.gov.ua. Retrieved from http:// medstat.gov.ua/ukr/normdoc.html [in Russian].

4. Asuquo, M., Umoh, M. \& V. Nwagbara (2012). Penetrating abdominal trauma: experience in a teaching hospital, calabar, Southern Nigeria. International Journal of Clinical Medicine, 3, 426. 5. Como, J.J., Bokhari, F., \& Chiu, W.C. (2010). Practice management guidelines for selective nonoperative management of penetrating abdominal trauma. Journal of Trauma, 68, 21-33. 6. Demetriades, D., Hadjizacharia, P., \& Constantinou, C. (2006). Selective nonoperative management of penetrating abdominal solid organ injuries. Annals of Surgery, 244, 620-628.

7. Demetriades, D., Rabinowitz, B., \& Sofianos, C. (2008). The management of penetrating injuries of abdomen. A prospective
4. Применение лапароскопии в диагностике и лечении колото-резаных ранений брюшной стенки является приоритетным методом в случае стабильной гемодинамики у больных [8].

8. Koto M. The role of laparoscopy in penetrating abdominal trauma: our initial experience / M. Koto, O. Matsevych, S. Motilall // Journal of Laparoendoscopic \& Advanced Surgical Techniques. - 2015. - №25. - P.730.

9. Mahmood I. Significance of computed tomography finding of intra-abdominal free fluid without solid organ injury after abdominal trauma: time for laparotomy on demand / I. Mahmood, Z. Tawfek, Y. Abdelrahman // World Journal of Surgery. - 2014. - №38. - P.11-14.

10. Navsaria P.H. Non-operative management of abdominal stab wounds-an analysis of 186 patients / P. H. Navsaria, J. U. Berli, S. Edu // South Africa Journal of Surgery. - 2007. - №45. P. 128-132.

11. Nicholson K. Management of patients with evisceration after abdominal stab wounds / K. Nicholson, K. Inaba, D. Skiada // American Surgeon. - 2014. - № 80. - P. 984.

12. Udobi K. F. Role of ultrasonography in penetrating abdominal trauma: a prospective clinical study / K. F. Udobi, A. Rodriguez, W. C. Chiu // Journal of Trauma. - 2001. № 50. - P. 475-479.

13. Velmahos G. C. Selective nonoperative management in 1,856 patients with abdominal gunshot wounds: should routine laparotomy still be the standard of care? / G. C. Velmahos, D. Demetriades, K. G. Toutouzas // Annals of Surgery. - 2001. № 234. - P. 395-402.

14. William W. Non-operative Management in Penetrating Abdominal Trauma / W. William, M. D. Hope, T. J. Stanton // Emergency Medicine. - 2012. - № 43. - P. 190-195.

study of 230 patients. Annals of Surgery, 207, 72-74.

8. Koto, M., Matsevych, O., \& Motilall, S. (2015). The role of laparoscopy in penetrating abdominal trauma: our initial experience. Journal of Laparoendoscopic \& Advanced Surgical Techniques, 25, 730.

9. Mahmood, I., Tawfek, Z., \& Abdelrahman, Y. (2014). Significance of computed tomography finding of intra-abdominal free fluid without solid organ injury after abdominal trauma: time for laparotomy on demand. World Journal of Surgery, 38, 11-14.

10. Navsaria, P.H., Berli, J.U., \& Edu, S. (2007). Non-operative management of abdominal stab wounds-an analysis of 186 patients. South Africa Journal of Surgery, 45, 128-132.

11. Nicholson, K., Inaba, K., \& Skiada, D. (2014). Management of Patients with Evisceration after Abdominal Stab Wounds. American Surgeon, 80, 984.

12. Udobi, K.F., Rodriguez, A. \& Chiu, W. C. Role of ultrasonography in penetrating abdominal trauma: a prospective clinical study. Journal of Trauma, 50, 475-479.

13. Velmahos, G.C., Demetriades, D., \& Toutouzas, K.G. (2001). Selective nonoperative management in 1,856 patients with abdominal gunshot wounds: should routine laparotomy still be the standard of care? Annals of Surgery, 234, 395-402.

14. William, W., Hope, M.D., \& Stanton, T.J. Non-operative Management in Penetrating Abdominal Trauma. Emergency Medicine , 43, 190-195. 
V. V. GANJI, M. A. KUBRAK, A. I. RYLOV., M. B. DANYLUK, A. I. GLAZKOV

Zaporizhzhia State Medical University

City Clinical Hospital of Emergency and First Medical Aid of Zaporizhzhia

\section{A UNIFIED APPROACH TO THE TREATMENT OF PATIENTS WITH PENETRATING STAB WOUNDS TO THE ABDOMEN}

During the period from 2010 to 2015, according to the Ministry of Health of Ukraine, the total mortality rate in Ukraine is at the level of $14.5-16.3 \%$. In $6 \%$ of all deaths the cause was trauma. Open penetrating abdominal trauma remains one of the major causes of deaths. While stab wounds are found almost three times more often than gunshot [3]. In recent years, the world has redefined approaches to the management of patients with penetrating wounds, leaning in the direction of selective use of operative and nonoperative methods of treatment [2].

Widespread is a method of selective non-operative management of patients with penetrating wounds of the abdomen $[4,10,14]$. The essence of this method is that by applying modern diagnostic methods, and clinical monitoring of the patient, in certain cases it is possible to dispense with the use of laparotomy. This technique allows in $55 \%$ of cases, stab wounds manage cases without resorting to surgical intervention, to reduce the number of unnecessary laparotomies to 9-19\%, reduce the duration of patients' stay in hospital was 3.9 days compared to 6, with laparotomy, as well as to reduce material costs for treatment of patients.

Selection of patients for non-operative management to better focus on the data of common clinical examination (overall sensitivity and specificity of serial clinical examination of the abdomen is 87.3 and $93.5 \%$ per cent respectively) [10]. A good help is the data of computer tomography, however, the sensitivity and specificity of this method ranges from different authors from $60 \%$ to over $90 \%[4,9,11,13]$. The diagnostic value of ultrasound examination in the first hours after the injury is just $46 \%$ [12], and review x-rays to $2.6 \%$ [7]. The use of laparoscopy in the diagnosis and treatment of stab wounds of the abdominal wall is a priority method in the case of stable hemodynamic patients [8].

Key words: selective non-operative management; stab wounds; abdominal cavity; laparoscopy.

\section{В. В. ГАНЖИЙ, М. А. КУБРАК, А. І. РИЛОВ, М. Б. ДАНИЛЮК, А. І. ГЛАЗКОВ}

Запорізький державний медичний університет

кУ "Міська клінічна лікарня екстреної та швидкої медичної допомоги м. Запоріжжя"

\section{УНІФІКОВАНИЙ ПІДХІД ДО ЛІКУВАННЯ ХВОРИХ ІЗ ПРОНИКАЮЧИМИ КОЛОТО-РІЗАНИМИ ПОРАНЕННЯМИ ЧЕРЕВНОЇ ПОРОЖНИНИ}

За період з 2010 до 2015 р., за даними МОЗ України, загальний показник смертності в Україні перебуває на рівні 14,5-16,3 \%. При цьому в 6 \% всіх випадків смерті причиною були травми. Відкрита проникаюча травма живота залишається однією з основних причин летальних випадків. Втім ножові поранення зустрічаються майже в три рази частіше, ніж вогнепальні [3]. В останні роки в світі переглянули підходи до ведення хворих із проникаючими пораненнями, схилившись у бік вибіркового застосування операційного і неопераційного методів лікування [2].

Значного поширення набув метод селективного неопераційного лікування хворих з проникаючими пораненнями черевної порожнини $[4,10,14]$. Суть даного методу полягає в тому, що, застосовуючи сучасні діагностичні методи, а також клінічний моніторинг стану хворого, в певних випадках можна обійтися без застосування лапаротомії. Дана методика дозволяє в 55 \% випадків ножових поранень вести хворих, не вдаючись до операційного втручання, скоротити кількість непотрібних лапаротомій до 9-19 \%, зменшити тривалість перебування хворих у стаціонарі до 3,9 дня порівняно з 6 при лапаротомії, а також знизити матеріальні витрати на лікування пацієнтів.

При відборі хворих для неопераційного ведення краще орієнтуватися на дані загальноклінічних досліджень (загальна чутливість і специфічність серійного клінічного обстеження черевної порожнини становить 87,3 і 93,5 \% відповідно) [10]. Доброю допомогою є дані комп’ютерної томографії, однак чутливість і специфічність даного методу коливається у різних авторів від 60 до 90 \% $[4,9,11,13]$. Діагностична цінність ультразвукового дослідження в перші години після отриманої травми становить всього 46 \% [12], а оглядової рентгенографії - 2,6 \% [7]. Застосування лапароскопії в діагностиці і лікуванні колото-різаних поранень черевної стінки є пріоритетним методом у разі стабільної гемодинаміки у хворих [8].

Ключові слова: селективне неопераційне ведення; ножові поранення; черевна порожнина; лапароскопія.

Получено 12.01.17 